\title{
Neuron firing in driven nonlinear integrate-and-fire models
}

\author{
Marcin Kostur, Michael Schindler, Peter Talkner * Peter Hänggi \\ Institut für Physik, Universität Augsburg, D-86135 Augsburg, Germany
}

Received 22 May 2006; received in revised form 11 August 2006; accepted 21 August 2006

Available online 25 August 2006

\begin{abstract}
Statistical properties of neuron firing are studied in the framework of a nonlinear leaky integrate-and-fire model that is driven by a slow periodic subthreshold signal. The firing events are characterized by first passage time densities. The experimentally better accessible interspike interval density generally depends on the sojourn times in a refractory state of the neuron. This aspect is not part of the integrate-and-fire model and must be modelled additionally. For a large class of refractory dynamics, a general expression for the interspike interval density is given and further evaluated for the two cases with an instantaneous resetting (i.e. no refractory state) and a refractory state possessing a deterministic lifetime. First passage time densities and interspike interval densities following from the proposed theory compare favorably with precise numerical simulations. (C) 2006 Elsevier Inc. All rights reserved.
\end{abstract}

PACS: 87.19.La; 05.40.-a; 02.70.Rr; 89.75.Hc; 82.20.Uv

Keywords: Driven nonlinear neuron models; Time dependent rates; Waiting time density; Interspike interval density; Refractory periods

\section{Introduction}

The firing activity of single neurons constitutes a basic aspect of any neuronal information processing and transmission. It still provides challenging problems and this is despite a very large number of works have been devoted to this objective [1-4].

\footnotetext{
${ }^{*}$ Corresponding author. Tel.: +49 821598 3233; fax: +49 8215983222.

E-mail address: Peter.Talkner@Physik.Uni-Augsburg.DE (P. Talkner).
} 
The theoretical description of single neuron dynamics has been attempted in the framework of different models, such as the classical Hodgkin-Huxley, the FitzHugh-Nagumo and the leaky integrate-and-fire model. Whereas the Hodgkin-Huxley model is based on the conductance properties of the neuron cell membrane resulting from the nature of sodium and potassium ion channels, the FitzHugh-Nagumo model is less detailed. It only contains the dynamics of the membrane voltage coupled to a so-called refractory variable which brings back the membrane voltage to its rest state after the neuron has fired. Even simpler is the leaky integrate-and-fire model, which describes the dynamics of the membrane voltage as a random motion under the influence of a leak current, which is linear in the membrane voltage, until a prescribed threshold is reached. This event causes the firing of the neuron. After firing, the membrane voltage is reset to its rest value.

The leaky integrate-and-fire model has been considered as an approximation of both the Hodgkin-Huxley [5] and the FitzHugh-Nagumo model [6]. In both cases, however, the resulting total current is nonlinear in the voltage with three fixed points where the current vanishes, see Fig. 1. Two of them are attractive and the one in between is repulsive. The smaller of the two stable voltage values is considered as the resting voltage. When the voltage increases beyond the unstable fixed point and reaches the stable fixed point with the larger voltage, the neuron fires and is set back to the resting voltage. The last step may require some time during which the neuron is in a refractory state. Being there the neuron is insensitive to the signal and may not fire.

Nonlinear integrate-and-fire models have been considered in different regimes. These can roughly be categorized by three criteria; whether they are deterministic or stochastic, whether the applied signal is static or time dependent, and whether this is a sub- or suprathreshold signal, i.e. whether the signal may drive the membrane voltage above the threshold in absence of random noise. No single analytic method is known that would yield reliable results in all different regimes. Either numerical methods have to be used or in specific cases, tailored approximations may be applied in order to analyze relevant aspects of the dynamical behavior of the model $[7-9,4]$. The numerical methods either deal with the stochastic differential equations $[10,11]$ or the equivalent Fokker-Planck equation [11-13]. For time dependent Ornstein-Uhlenbeck processes the first passage time density can also be obtained from an integral equation [14], which provides a convenient starting point for numerical investigations [15,13].

In the present work we will consider an integrate-and-fire model with a cubic nonlinearity as was suggested by Tuckwell [1] driven by a subthreshold periodic signal with a long period compared to the characteristic relaxation time towards the resting voltage. The small random contri-

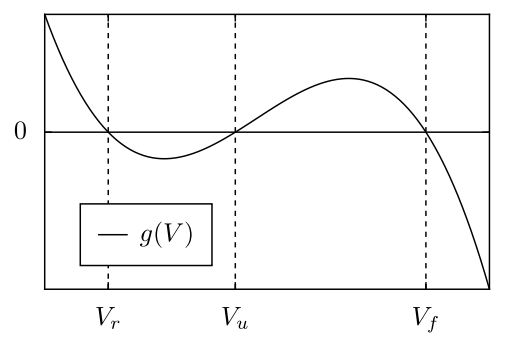

Fig. 1. Schematic view of a typical neuronal current-voltage characteristics. The current vanishes at the resting voltage $V_{\mathrm{r}}$, the unstable voltage $V_{\mathrm{u}}$ and the firing voltage $V_{\mathrm{f}}$, see also the description of the model in Section 2. 
bution to the dynamics of the membrane voltage is represented by Gaussian white noise, which only rarely drives the voltage to the threshold. A similar model with a bistable force was discussed by Chow and White [5] for small noise under constant subthreshold forcing. On a time scale which is long compared to both the typical relaxation time toward the resting voltage and typical refractory times the interspike interval times, or residence times, i.e. the times between adjacent firing events, are exponentially distributed. The respective rate was determined by Chow and White [5] as the Kramers rate [16] describing the noise activated escape from the resting state. Other nonlinear integrate-and-fire models as e.g. described by Fourcaud-Trocmé et al. [17] can be analyzed by the method presented here.

The envisaged case though is more complicated due to the time dependence of the driving signal. For the slow subthreshold driving considered here we may follow the line of reasoning that was applied to the linear leaky integrate-and-fire model [12,13] and outlined in Ref. [18] in more detail. A related consideration is found in Ref. [19] for a bistable periodically driven system. The key point is that the essential information about the long time dynamics is contained in an escape rate, which is time dependent as a consequence of the time dependent signal. This rate at first determines the waiting time distribution in the resting state and consequently the first passage time density, i.e. the probability density of the very time at which the firing state is reached for the first time. In the case of periodic driving, the waiting time density and the first passage time density depend on the phase of the driving force at the starting time. In order to find the interspike interval density the first passage time density has to be averaged over the starting times resulting from firing times [20-22] and refractory periods. If the refractory periods last only short time compared to the typical interspike interval times the density of firing times is given by the firing rate [23]. Therefore, the primary task is to find the firing rate. For weak noise and slow driving frequencies the time dependent rates are given by the values of the rates at the instantaneous driving strength $[18,12,13,19]$.

The paper is organized as follows. In Section 2 we specify the model and the parameters to be considered. In Section 3 the relevant theory is reviewed and general expressions for the first passage time density and the interspike interval density are presented. In Section 4 we compare these theoretical results with the simulations for the first passage time density at a particular, fixed initial phase and of the interspike interval density. The paper ends with a resume given in Section 5.

\section{Nonlinear integrate-and-fire model}

In the nonlinear integrate-and-fire model the time evolution of the membrane voltage $V$ is given by a Langevin equation, reading

$$
C \frac{\mathrm{d} V}{\mathrm{~d} t^{\prime}}=g(V)+I\left(t^{\prime}\right)+\eta\left(t^{\prime}\right)
$$

where $C$ is the membrane capacitance per area, $g(V)$ the current-voltage characteristics of the membrane, $I\left(t^{\prime}\right)+\eta\left(t^{\prime}\right)$ the injected current resulting from an applied signal $I\left(t^{\prime}\right)$, which is contaminated by the noise source $\eta\left(t^{\prime}\right)$, and $t^{\prime}$ the time. Here we will restrict ourselves to periodic currents of the form

$$
I\left(t^{\prime}\right)=I_{0} \sin \Omega_{0} t^{\prime}
$$


with the amplitude $I_{0}$ and driving frequency $\Omega_{0}$. The random contribution to the current is modelled as Gaussian white noise; i.e.

$$
\left\langle\eta\left(t^{\prime}\right)\right\rangle=0, \quad\left\langle\eta\left(t^{\prime}\right) \eta\left(s^{\prime}\right)\right\rangle=2 D_{0} \delta\left(t^{\prime}-s^{\prime}\right) .
$$

According to the Hodgkin-Huxley model the noise strength $D_{0}$ is inversely proportional to the number of ion channels per area and, in principle, also a function of the membrane voltage, a dependence which we neglect here. In principle, other noise sources may also contribute to the random part of the current.

For the current-voltage characteristics we assume a cubic polynomial in the voltage with three real stationary points where the corresponding currents vanish, see Fig. 1,

$$
g(V)=\kappa\left(V-V_{\mathrm{r}}\right)\left(V-V_{\mathrm{u}}\right)\left(V-V_{\mathrm{f}}\right),
$$

where $\kappa$ is a positive constant. The smallest, stable stationary point $V_{\mathrm{r}}$ corresponds to the resting voltage, the middle one, $V_{\mathrm{u}}$ is unstable and the largest one, $V_{\mathrm{f}}$ is the voltage that causes the neuron to fire. For the shifted dimensionless voltage $x=\left(V-V_{\mathrm{r}}\right) /\left(V_{\mathrm{f}}-V_{\mathrm{r}}\right)$ we obtain the Langevin equation in terms of the rescaled dimensionless time $t=\kappa\left(V_{\mathrm{f}}-V_{\mathrm{r}}\right)\left(V_{\mathrm{u}}-V_{\mathrm{r}}\right) t^{\prime} / C$

$$
\dot{x}=-a^{-1} x(x-a)(x-1)+A \sin \Omega t+\sqrt{2 D} \xi(t),
$$

where $a=\left(V_{\mathrm{u}}-V_{\mathrm{r}}\right) /\left(V_{\mathrm{f}}-V_{\mathrm{r}}\right)$ is a positive parameter less than unity specifying the unstable state, $A=I_{0} / \kappa\left(V_{\mathrm{u}}-V_{\mathrm{r}}\right)\left(V_{\mathrm{f}}-V_{\mathrm{r}}\right)^{2}$ the dimensionless driving amplitude, $\Omega=C \Omega_{0} / \kappa\left(V_{\mathrm{u}}-V_{\mathrm{r}}\right)\left(V_{\mathrm{f}}-V_{\mathrm{r}}\right)$ the dimensionless frequency, $D=D_{0} / \kappa C\left(V_{\mathrm{u}}-V_{\mathrm{r}}\right)\left(V_{\mathrm{f}}-V_{\mathrm{r}}\right)^{3}$ the dimensionless noise strength and $\xi(t)$ Gaussian white noise with $\langle\xi(t) \xi(s)\rangle=\delta(t-s)$. The drift in the Langevin equation follows from the negative spatial derivative of a potential function $U(x, t)=x^{2} / 2-(a+1) x^{3} / 3 a+x^{4} /$ $4 a-A x \sin \Omega t$. We here consider subthreshold signals such that the potential $U(x, t)$ maintains its qualitative structure with two minima and a maximum for all times $t$. This requires that the signal amplitude $A$ stays below a critical value dependent on the parameter $a$. The positions of the extrema of $U(x, t)$ follow as the zeros of the deterministic drift, i.e.

$$
-a^{-1} x(x-a)(x-1)+A \sin \Omega t=0 .
$$

These time dependent values will be denoted as the instantaneous rest state $x_{\mathrm{r}}(t)$, unstable state $x_{\mathrm{u}}(t)$ and firing state $x_{\mathrm{f}}(t)$ taking the values $0, a$ and 1 , respectively, at time $t=0$. The values of the potential at the rest and unstable states are denoted by $U_{\mathrm{r}}(t)=U\left(x_{\mathrm{r}}(t), t\right)$ and $U_{\mathrm{u}}(t)=$ $U\left(x_{\mathrm{u}}(t), t\right)$, respectively. For later use, we introduce the potential curvatures at the rest and unstable states

$$
\omega_{\mathrm{r}}(t)^{2}=\left.\frac{\partial^{2} U(x, t)}{\partial x^{2}}\right|_{x=x_{\mathrm{r}}(t)} \quad \omega_{\mathrm{u}}(t)^{2}=-\left.\frac{\partial^{2} U(x, t)}{\partial x^{2}}\right|_{x=x_{\mathrm{u}}(t)} .
$$

We assume that the barrier height $\Delta U(t)=U_{\mathrm{u}}(t)-U_{\mathrm{r}}(t)$ of the potential, as seen from the rest state, is always considerably larger than the noise strength $D$. As a consequence of this assumption, the voltage will only rarely cross this potential barrier. The barrier crossings then are independent events, which consequently can be characterized by a firing rate $k(t)$. The time dependence of the rate results from the time dependence of the signal. 


\section{Firing time statistics}

Finally, we assume that the period of the signal $T=2 \pi / \Omega$ is long compared to the relaxation time towards the rest state, i.e. with the present scaling $T \gg 1$. Then, the time dependent firing rate can be determined in an adiabatic way as the frozen rate out of the respective potential well $U(x, t)$. This will be done in the next subsection. We note that the adiabatic approximation is only employed to determine the rate. Because the resulting rate may be smaller than or comparable to the driving frequency $\Omega$ the long time dynamics, which is induced by this rate, need not instantaneously follow the applied signal. The combination of the adiabatic rate with a nonadiabatic rate dynamics characterizes the semi-adiabatic regime $[18,24]$

\subsection{Firing rate}

The rate, which describes the frequency of transitions per unit time in the frozen potential from the resting state to the firing state, is approximated by the Kramers rate expression [16]

$$
k(t)=\frac{\omega_{\mathrm{r}}(t) \omega_{\mathrm{u}}(t)}{2 \pi} \mathrm{e}^{-\Delta U(t) / D} .
$$

This expressions holds with a relative error of less than $7 \%$ if the barrier height $\Delta U(t)$ is at least $6 D$. For lower barriers a more involved, exact rate expression can be given in terms of the inverse mean first passage time from the resting to the firing state in the frozen potential [16]. It turns out that the mean first passage time is rather insensitive regarding the precise location of the starting and target states as long as they are sufficiently remote from the unstable state and the barrier height is larger than $2 D$. If the latter condition is not met the time scale separation between fast intra-well relaxation and rare inter-well transitions gets lost, the mean first passage time does depend on the detailed starting and target conditions and consequently a Markovian rate description of the firing process loses validity.

\subsection{Densities of first passage times, firing times and interspike intervals}

Within the above specified rate regime the probability $P(t \mid s)$ that the neuron has not fired up to time $t$ under the condition that it had been started in the rest state at the previous time $s$ follows from the rate equation

$$
\begin{aligned}
\frac{\partial}{\partial t} P(t \mid s) & =-k(t) P(t \mid s) \quad \text { for } t>s, \\
P(s \mid s) & =1
\end{aligned}
$$

to read

$$
P(t \mid s)=\exp \left\{-\int_{s}^{t} \mathrm{~d} s^{\prime} k\left(s^{\prime}\right)\right\} \text { for } t \geqslant s .
$$

The first passage time density $g(t \mid s)$ then results from the negative derivative of $P(t \mid s)$ with respect to $t$ 


$$
g(t \mid s)=-\frac{\partial}{\partial t} P(t \mid s)=k(t) \exp \left\{-\int_{s}^{t} \mathrm{~d} s^{\prime} k\left(s^{\prime}\right)\right\} \text { for } t \geqslant s .
$$

From an experimental point of view it would be rather difficult to determine this first passage time density $g(t \mid s)$ because it would require to condition the neuron in the resting state at a prescribed phase of the signal [25]. In the experiment it is easier to observe the interspike interval density characterizing the statistics of the time intervals between consecutive firing events. This statistics is given by an appropriate average over the first firing time density. In general, this average though depends on a possibly existing refractory period after each firing event during which the neuron is not able to fire. In a first step we disregard this case and assume that after the neuron has fired it immediately is reset into the resting state. Then each firing event fixes the starting instant for the next interspike interval. Hence, the interspike interval density is an average of $g(\tau+s \mid s)$ over the properly normalized density of firing events within a period of the driving force. The not normalized density of firing events is given by the product of the firing rate $k(s)$ and the probability $p_{\mathrm{f}}(t)$ to find the neuron in the rest state ready to fire [23]. In the absence of a refractory period this probability is unity, $p_{\mathrm{f}}(t)=1$. Therefore one obtains for the interspike interval density [12]

$$
h(\tau)=\frac{\int_{0}^{T} \mathrm{~d} s g(\tau+s \mid s) k(s)}{\int_{0}^{T} \mathrm{~d} s k(s)},
$$

where $T=\Omega / 2 \pi$ denotes the signal period. The term in the denominator takes care of the normalization of the firing time density.

\subsection{Refractory times and interspike intervals}

In contrast to the more detailed Hodgkin-Huxley and FitzHugh-Nagumo models, integrateand-fire models do not provide information about possible refractory periods. Therefore additional assumptions are necessary to include this aspect into this class of models. We base our treatment on the following hypotheses: (i) The neuron stays in a refractory state for some time after each firing event. From the refractory state it goes into the rest state from where it is ready to fire again according to the first passage time density $g(t \mid s)$. Here the starting time $s$ is given by the time of the last transition from the refractory to the rest state. The sojourn times in this refractory state are statistically independent of (ii) the signal, (iii) the previous sojourn times, and (iv) the history of the previous firing events. Consequently, the refractory state can fully be characterized by the refractory time density $\rho_{\text {ref }}(t)$ to find a refractory period of length $t$. Finally we require that (v) the refractory time density has a finite mean value which is short compared to the shortest inverse rate, i.e. $\int_{0}^{\infty} t \rho_{\text {ref }}(t) \mathrm{d} t \ll \min _{t} k(t)^{-1}$. The first four assumptions imply that the two state process of consecutive refractory and rest states is a continuous time random walk [26]. The probability $p_{\mathrm{f}}(t)$ that at time $t$ this process is in the rest state follows from the solution of the corresponding generalized master equation [26], which we will not discuss here. Then the interspike interval density becomes

$$
h(\tau)=\frac{\int_{0}^{T} \mathrm{~d} t \int_{0}^{\infty} \mathrm{d} s g(t+\tau \mid t+s) \theta(\tau-s) \rho_{\text {ref }}(s) k(t) p_{\mathrm{f}}(t)}{\int_{0}^{T} \mathrm{~d} t k(t) p_{\mathrm{f}}(t)} .
$$


In the integral the heaviside function $\theta(t)$ ensures that the firing time $t+\tau$ in the first passage time density $g(t+\tau \mid t+s)$ never precedes the starting time $t+s$. Under the assumption (v) the rest state is realized almost certainly and hence $p_{\mathrm{f}} \approx 1$ as in the absence of a refractory period. Then the interspike interval density simplifies to read

$$
h(\tau)=\frac{\int_{0}^{T} \mathrm{~d} t \int_{0}^{\infty} \mathrm{d} s g(t+\tau \mid t+s) \theta(\tau-s) \rho_{\mathrm{ref}}(s) k(t)}{\int_{0}^{T} \mathrm{~d} t k(t)} .
$$

In the case of a deterministic refractory time $t_{\text {ref }}$, i.e. for $\rho_{\text {ref }}(s)=\delta\left(s-t_{\text {ref }}\right)$ the interspike interval density becomes

$$
h(\tau)=\frac{\int_{0}^{T} \mathrm{~d} t g\left(t+\tau \mid t+t_{\mathrm{ref}}\right) k(t)}{\int_{0}^{T} \mathrm{~d} t k(t)} \theta\left(\tau-t_{\mathrm{ref}}\right) .
$$

Clearly, this density vanishes for times $\tau$ that are shorter than the refractory times. For a vanishing refractory time $t_{\text {ref }}=0$ the previous result (12) is recovered.

\section{Comparison with numerical simulations}

We numerically implemented the Langevin equation (Eq. 5) with explicit time steps of width $h=0.01$. We chose $a=0.4$ for the value of the rescaled unstable voltage, $A=0.01$ and $\Omega=0.01$ for the signal amplitude and frequency, respectively, and $D=0.003$ for the noise strength. The resulting ratio of barrier height and noise strength, $\Delta U(t) / D$ varies between the approximate limits 6 and 9. In this regime the time scales of intra-well relaxations and escape times are separated by 3-4 orders of magnitude, hence, a rate description of the long time dynamics is justified. With $\Omega=0.01$ the signal is much faster than the escape but still sufficiently slow compared to the intra-well relaxation rate, which is 1 in the used dimensionless units, such that the frozen Kramers rates can be expected to apply. We estimated the first passage time density from an ensemble of $5 \times 10^{4}$ simulated Langevin trajectories. Each trajectory was started with the same signal phase at $t=0$ at the rest state $x=0$ and stopped when it had reached the value $x=0.757$. The probability of an immediate recrossing of the barrier from there is less than $3 \times 10^{-6}$ [27] and therefore may safely be neglected. On the other hand, the time to approach the final firing state $x_{\mathrm{f}}$ from this threshold is of the order one and therefore negligibly short compared to the typical time needed to reach the threshold. The times when the threshold was reached were collected in a histogram and are displayed in Fig. 2.

For the simulation of the interspike interval density a single trajectory suffices. Because it may contain long episodes of many driving periods without firing events this trajectory has to be quite long to produce a reliable interval statistics. We started in the rest state at time $t=0$ until the threshold was reached for the first time $t_{\mathrm{f} 1}$. In absence of a refractory period, the re-scaled potential then was reset to the rest state $x_{\mathrm{r}}\left(t_{\mathrm{f}}\right)$ and the process was run with the continuing signal left unaffected by the firing process until the threshold was reached again at a time $t_{\mathrm{f} 2}$. This procedure was repeated until $10^{5}$ firing events had occurred. We collected the interspike intervals $t_{\mathrm{f} n+1}-t_{\mathrm{f} n}$ in a histogram which serves as an estimator for the corresponding probability density, see Fig. 3. 


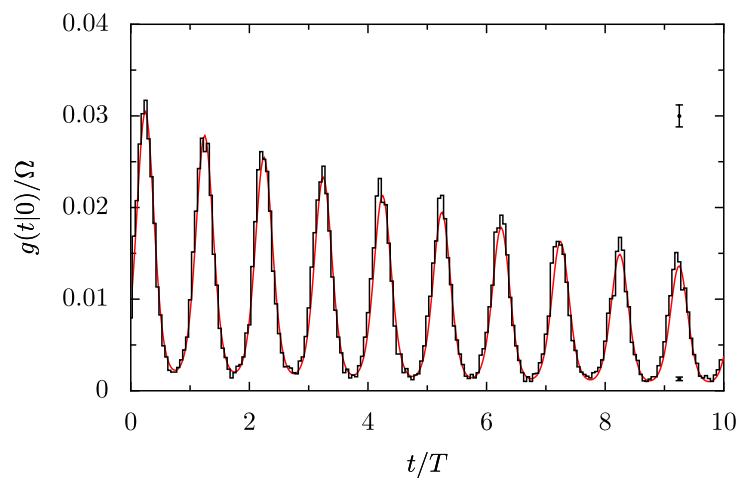

Fig. 2. The histogram (steplike graph) of first passage times resulting from $10^{5}$ simulated trajectories of the nonlinear leaky integrate-and-fire model (5) is compared with the first passage time density $g(t, 0)$ given by Eq. (11) (smooth curve) as a function of firing time $t$ in units of the driving period $T=2 \pi / \Omega$ for the parameter values $a=0.4, A=0.01$, $D=0.003$. The simulated trajectories start at time $t=0$ at $x=0$ and are run until the value $x=0.757$ is reached. The two bars on the right side of the figure indicate the largest and smallest occurring statistical errors of the histogram for density values 0.03 and 0.002 , respectively. There are no significant deviations between simulations and theory. The multi-peaked density slowly decays with the average rate $k_{\mathrm{a}}=\int_{0}^{T} \mathrm{~d} t k(t) / T$. Superimposed on this decay is a periodic function with the period $T$ of the driving signal.

Finally we estimated the interval interspike density in presence of a deterministic refractory period of length $t_{\text {ref }}=T / 2$. We chose this long refractory period in order to obtain a clearly visible effect. In this case we proceeded analogously as in the previous case without refractory period except that after a firing event at a time $t_{\mathrm{f}}$ we restarted the process only at the later time $t_{\mathrm{f}}+t_{\text {ref }}$ with the continually running signal phase $\Omega\left(t_{\mathrm{f}}+t_{\text {ref }}\right)$. The corresponding histogram of $10^{5}$ firing events is compared with the theoretical result, Eq. (15), in Fig. 3.

\section{Conclusions}

We determined the statistics of firing events in terms of the first passage time density and the interspike interval density for a nonlinear leaky integrate-and-fire model with a cubic currentvoltage characteristics, which in addition is driven by a periodic subthreshold signal. The random contribution to the current was assumed to be so small that noise induced transitions from the resting to the firing state presented rare events. The signal period was chosen long compared to the local relaxation time in the resting state. Under these conditions the long time dynamics of the resting state is Markovian and consequently characterized by a rate which is time-dependent due to the presence of the time dependent signal.

In order to model the influence of a refractory period we have assigned an extra state to this period which is characterized by a waiting time distribution such that the sequence of refractory and resting states constitute a continuous time random walk, or in other words, a semi-Markov process.

The agreement between the theory and the numerically precise simulations of the underlying Langevin equation dynamics describing the leaky integrate-and-fire model turns out to be very good for both the first passage time density and the interspike interval density. This good agreement holds true also for the two cases with and without a deterministic refractory period. 

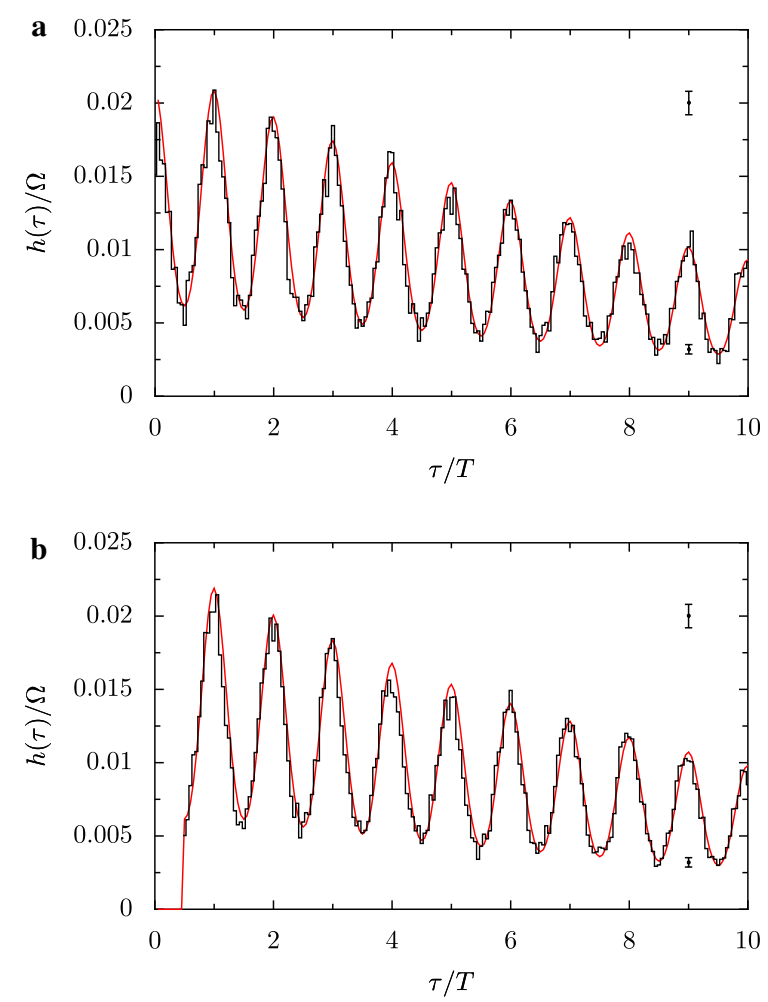

Fig. 3. The histogram (steplike graph) of the interspike intervals resulting from a simulation of the nonlinear leaky integrate-and-fire model (5) is compared with the interspike interval density $h(t)$ given by Eq. (15) (smooth curve) as a function of the interval duration in units of the driving period $T=2 \pi / \Omega$ for the parameter values $a=0.4, A=0.01$, $D=0.003$. The two bars on the right side indicate the largest and smallest occurring statistical errors of the histogram for the density values 0.02 and 0.005 , respectively. There are no significant deviations between simulations and theory. In the upper panel (a), the refractory time $t_{\mathrm{f}}$ is zero and in the lower panel (b) it coincides with half the period, $t_{\text {ref }}=T / 2$. Most evidently, a finite deterministic refractory time suppresses the immediate succession of firing events. Upon closer inspection one finds the probability which is removed by the presence of the refractory state from the short intervals transferred to the longer ones. Local maxima of the interspike interval density are found at integer multiples of the signal period.

\section{Acknowledgments}

The authors thank Dr. Igor Goychuk and Dr. Gerhard Schmid for valuable discussions. This work was supported by the Deutsche Forschungsgemeinschaft via the project HA1517/13-4 and SFB-486, Projects A10 and B13 and the Volkswagen Foundation, via project No. I/80424.

\section{References}

[1] H.C. Tuckwell, Stochastic Processes in the Neurosciences, SIAM, Philadelphia, 1989.

[2] L.M. Ricciardi, Diffusion Processes and related topics in Biology, Springer, Berlin, 1977. 
[3] L.M. Ricciardi, S. Sato, Diffusion processes and first passage time problems, in: L.M. Ricciardi (Ed.), Lectures in Applied Mathematics and Informatics, Manchester University Press, Manchester, 1990, p. 206.

[4] B. Lindner, J. Garcia-Ojalvo, A. Neiman, L. Schimansky-Geier, Effects of noise in excitable systems, Phys. Rep. $392(2004) 321$.

[5] C.C. Chow, J.A. White, Spontaneous action potentials due to channel fluctuations, Biophys. J. 11 (1996) 3013.

[6] H.C. Tuckwell, R. Rodriguez, F.Y.M. Wan, Determination of firing times for stochastic Fitzhugh-Nagumo neuronal model, Neural Comp. 15 (2003) 143.

[7] M.A. Zaks, X. Sailer, L. Schimansky-Geier, A.B. Neimann, Noise induced complexity: from subthreshold oscillations to spiking in coupled excitable systems, Chaos 15 (2005) 026117.

[8] L. Gammaitoni, P. Hänggi, P. Jung, F. Marchesoni, Stochastic resonance, Rev. Mod. Phys. 70 (1998) 223.

[9] P. Hänggi, Stochastic resonance in biology - How noise can enhance detection of weak signals and help improve biological information processing, Chem. Phys. Chem. 3 (2002) 285.

[10] M.T. Giraudo, L. Sacerdote, Simulation methods in neuronal modelling, BioSystems 48 (1998) 77.

[11] N. Fourcaud, N. Brunel, Dynamics of the firing probability of integrate-and-fire neurons, Neural Comput. 14 (2002) 2057.

[12] M. Schindler, P. Talkner, P. Hänggi, Firing time statistics for driven neuron models: analytic expressions versus numerics, Phys. Rev. Lett. 93 (2004) 048102.

[13] M. Schindler, P. Talkner, P. Hänggi, Escape rates in periodically driven Markov processes, Physica A 351 (2005) 40.

[14] A. Buonocore, A.G. Nobile, L.M. Ricciardi, A new integral equation for the evaluation of 1st passage time probability densities, Adv. Appl. Probab. 19 (1987) 784.

[15] E. Di Nardo, A.G. Nobile, E. Pirozzi, L.M. Ricciardi, A computational approach to first passage time problems for Gauss-Markov processes, Adv. Appl. Probab. 33 (2001) 453.

[16] P. Hänggi, P. Talkner, M. Borkovec, Reaction-rate theory: fifty years after Kramers, Rev. Mod. Phys. 62 (1990) 251.

[17] N. Fourcaud-Trocmé, D. Hansel, C. van Vresswijk, N. Brunel, How spike generation mechanisms determine the neuronal response to fluctuating inputs, J. Neurosci. 23 (2003) 11628.

[18] P. Talkner, J. Łuczka, Rate description of Fokker-Planck processes with time-dependent parameters, Phys. Rev. E 69 (2004) 046109.

[19] P. Talkner, L. Machura, M. Schindler, P. Hänggi, J. Łuczka, Statistics of transition times, phase diffusion and synchronization in periodically driven bistable systems, New J. Phys. 7 (2005) 14.

[20] R. Löfstedt, S.N. Coppersmith, Stochastic resonance-Nonperturbative calculation of power spectra and residence time distributions, Phys. Rev. E 49 (1994), 4821.

[21] M.H. Choi, R.F. Fox, P. Jung, Quantifying stochastic resonance in bistable systems: response vs residence-time distribution functions, Phys. Rev. E 57 (1998) 6335.

[22] T. Shimokawa, K. Pakdaman, T. Takahata, S. Tanabe, S. Sato, A first-passage-time analysis of periodically forced the leaky integrate-and-fire model, Biol. Cybern. 83 (2000) 327.

[23] P. Talkner, Statistics of entrance times, Physica A 325 (2003) 124.

[24] P. Talkner, Stochastic resonance in the semiadiabatic limit, New J. Phys. 1 (1999) 4.

[25] P. Lánský, Sources of periodical force in noisy integrate-and-fire models of neuronal dynamics, Phys. Rev. E 55 (1997) 2040.

[26] E.W. Montroll, H. Scher, Random walks on Lattices. IV. Continuous-time walks and influence of absorbing boundaries, J. Stat. Phys. 9 (1973) 101.

[27] This value follows from the frozen splitting probability to first reach the resting voltage 0 , rather than the firing voltage if one starts out in $x$. It is given by $\pi(x, t)=\int_{x}^{1} \exp \{U(x, t) / D\} / \int_{0}^{1} \exp \{U(x, t) / D\}[28]$.

[28] N.G. van Kampen, Stochastic Processes in Physics and Chemistry, North Holland, Amsterdam, 1992. 